\title{
Technè
}

La science au service de l'histoire de l'art et de la préservation des biens culturels

$40 \mid 2014$

Thérapéia. Polychromie et restauration de la sculpture dans l'Antiquité

\section{(Re)peindre, dorer, cirer. La thérapéia en acte dans la sculpture grecque hellénistique}

(Re)painting, gilding, waxing. The process of therapeia in Hellenistic Greek sculpture

\section{Brigitte Bourgeois}

\section{OpenEdition \\ Journals}

Édition électronique

URL : https://journals.openedition.org/techne/3651

DOI : 10.4000/techne.3651

ISSN : 2534-5168

\section{Éditeur}

C2RMF

Édition imprimée

Date de publication : 26 novembre 2014

Pagination : 69-80

ISBN : 978-2-7118-6218-4

ISSN : $1254-7867$

\section{Référence électronique}

Brigitte Bourgeois, « (Re)peindre, dorer, cirer. La thérapéia en acte dans la sculpture grecque

hellénistique », Technè [En ligne], 40 | 2014, mis en ligne le 24 juillet 2020, consulté le 08 février 2022.

URL : http://journals.openedition.org/techne/3651 ; DOI : https://doi.org/10.4000/techne.3651

\section{(c) $(1)$}

La revue Technè. La science au service de l'histoire de l'art et de la préservation des biens culturels est mise à disposition selon les termes de la Licence Creative Commons Attribution - Pas d'Utilisation Commerciale - Pas de Modification 4.0 International. 
Brigitte Bourgeois

\section{(Re)peindre, dorer, cirer. La thérapéia en acte dans la sculpture grecque hellénistique}

(Re)painting, gilding, waxing. The process of therapeia in Hellenistic Greek sculpture

«Considérez donc ces statues qui semblent respirer, dont vous touchez et effleurez les pieds et les genoux en les priant: ne voyez-vous pas que tantôt elles s'effritent sous les gouttes de pluie, tantôt elles se désintègrent sous la pourriture qui les ronge; qu'exposées à la fumée des parfums, elles perdent leurs couleurs et noircissent, et qu'enfin, lorsqu'elles ont été laissées trop longtemps sans soins et à l'abandon, elles perdent leur aspect et se dégradent sous l'effet de la rouille qui les dévore?»

Arnobe, Adversus nationes, VI, 16, 6, trad. B. Fragu, Paris, Les Belles Lettres, 2010.

Résumé. Expression de piété, la thérapéia, le "service » rendu aux effigies sacrées dans la Grèce antique, désignait dans un sens profane des opérations liées à la conservation des xuvres, visant à remettre en bon ordre la statue aux mains d'artisans choisis pour leur savoir-faire. L'article présente trois dossiers de sculptures en marbre d'époque hellénistique qui permettent d'approcher concrètement la réalité des gestes opérés en matière de traitements de surface tels que peindre, nettoyer, repeindre, dorer, ou encore appliquer un vernis de cire d'abeille selon l'opération de la ganôsis. Les études de cas concernent une statuette d'Asclépios (Dresde), la statuette féminine dorée de la Maison des Masques (Délos) et le portrait dit de Bérénice II (Musée royal de Mariemont).

Mots-clés. Polychromie, Grèce, antiquité, marbre, vidéomicroscopie, microscopie 3D de surface, kosmèsis, nettoyage, repeint, dorure, blanc de plomb, ganôsis, cire d'abeille, Égypte ptolémaïque, Bérénice II, Treu, Délos, Mariemont.

\begin{abstract}
An expression of piety, therapeia - the "service" rendered to sacred effigies in Ancient Greece - meant, in a secular sense, conservation-related actions, whose purpose was to restore statues to good condition and which were carried out by craftsmen chosen for their savoir-faire. This article presents three studies of marble sculptures dating from the Hellenistic period that have given us concrete knowledge about the actual gestures used in surface treatments such as painting, cleaning, repainting, gilding or even applying a beeswax varnish in the process called ganosis. The case studies here concern a statuette of Asclepios (Dresden), the gilded female statuette from the House of the Masks (Delos) and the portrait known as Berenice II (Musée royal de Mariemont, Belgium).
\end{abstract}

Keywords. Polychromy, Greece, Antiquity, marble, video microscopy, 3D surface microscopy, kosmesis, cleaning, repaint, gilding, lead white, ganosis, beeswax, Ptolemaic Egypt, Berenice II, Treu, Delos, Mariemont.

\section{Introduction : «l'idole des origines »}

Au cours du long processus de redécouverte des couleurs de l'Antique, initié au XVIII ${ }^{\mathrm{e}}$ siècle, les restes de polychromie détectés sur des marbres, qu'il s'agisse de sculpture ou d'architecture, ont été en général rapportés au temps de la création et considérés comme les vestiges d'un état premier. L'interprétation, d'ailleurs en partie fondée ou plausible, s'inscrivait tout naturellement au cœur d'une démarche archéologique conçue comme un dévoilement du passé le plus ancien. Dans le cas des études sur la Grèce ancienne, la quête des origines devait prendre une ampleur particulière en raison du phénomène singulier que constituait la disparition quasi-totale des chefs-d'œuvre de peinture et de sculpture dont parlait la littérature antique. Pour retracer le type et le style des originaux perdus, la recherche moderne s'est donc efforcée de définir ce que pouvait être la pureté première des commencements, celle du génie $d u$ «miracle grec », via la critique de copies souvent plus tardives et jugées décadentes. Fort de ce postulat, le «démon des origines", cette «idole de la tribu des historiens ${ }^{1}$ » contre laquelle Marc Bloch mettait en garde, a eu beau jeu de se déployer. Et la question du devenir de la création, la vision en quelque sorte évolutionniste d'œuvres nécessairement altérées et transformées au cours du temps ne pouvait que susciter un moindre intérêt. 


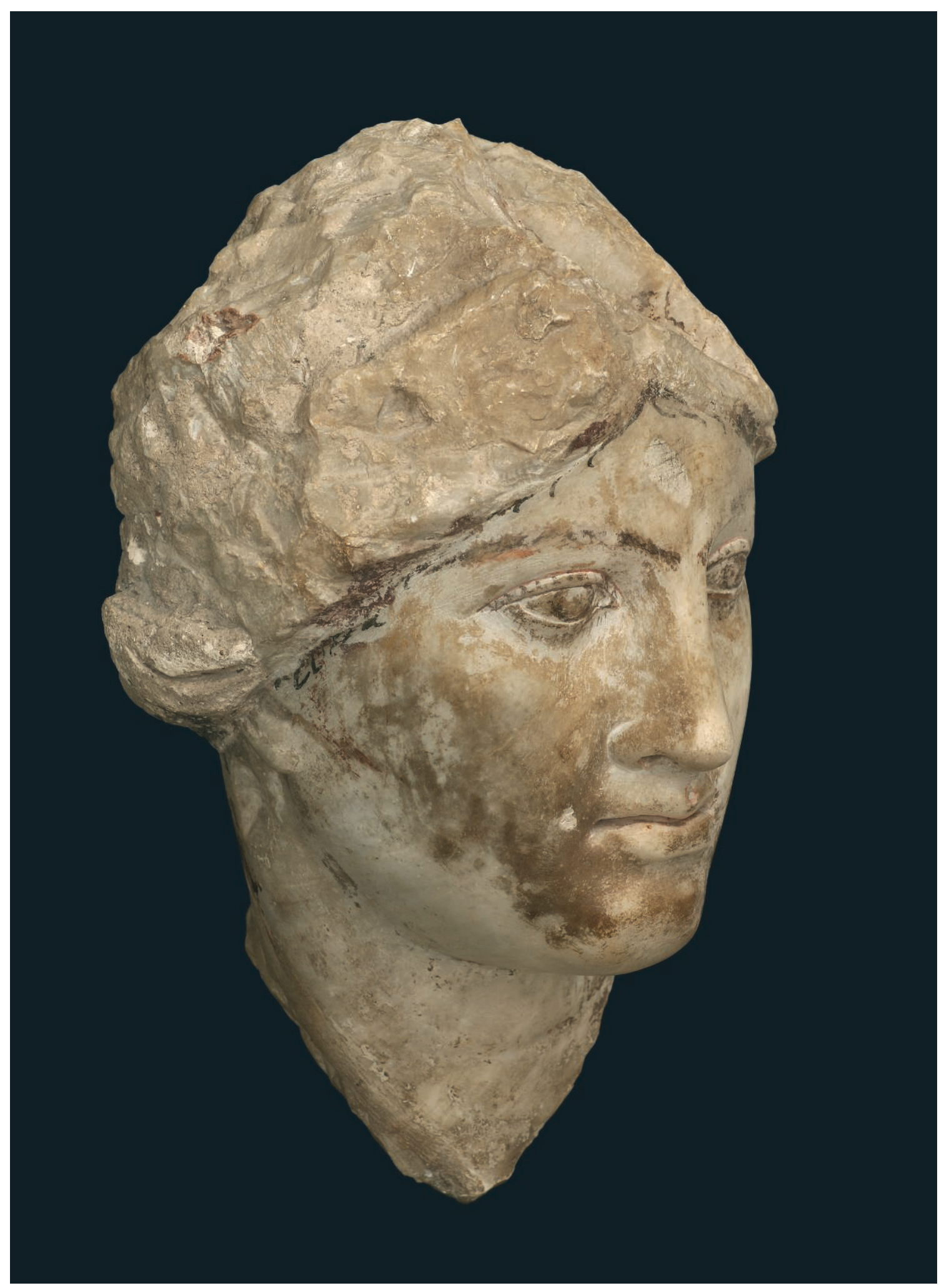

Fig. 1. Portrait de la reine Bérénice II, provenant

d'Égypte (Hermoupolis Magna), fin du III ${ }^{\mathrm{e}}$ siècle av. J.-C.,

H. $33 \mathrm{~cm}$, marbre blanc avec complément en stuc, restes

de peinture, de dorure et de cire, Musée royal de

Mariemont, inv. B. 264. @ Musée royal de Mariemont,

Michel Lechien. 
La tendance s'est depuis lors infléchie et la recherche ne cesse de se développer dans de nouvelles directions, qu'il s'agisse d'explorer les fonctions que l'objet a assumées durant sa «vie sociale», d'identifier les manipulations et réappropriations incessantes qu'il a connues ou bien d'interroger les notions de fondation, d'érosion et de transmission.

La question du devenir est évidemment cruciale pour une matière aussi fragile que la polychromie, sujette à se corrompre et destinée à être remaniée pour durer. Elle est au cœur de cet article qui vise à mettre en regard des sources textuelles l'évidence matérielle de reprises antiques détectées sur des effigies en marbre de l'époque hellénistique, notamment grâce à des examens que j'ai menés en vidéo-microscopie ${ }^{2}$. L'enquête - de "micro-terrain ", pourrait-on dire - cherche à faire l'archéologie de cette notion grecque de thérapéia dont traitent Evridiki Leka et Véronique Chankowski dans ce volume. Peindre, donc, mais aussi nettoyer et dépeindre, repeindre, dorer ou appliquer un vernis de cire, comme l'illustre le cas du portrait de Bérénice II (fig. 1), tels sont quelques-uns des gestes abordés ici. Ils permettront d'approcher plus concrètement, sur trois dossiers, la réalité des opérations de kosmèsis ou d'épikosmèsis, entendue au sens d'ornementation et de (re)mise en bon ordre de la statue, qui étaient confiées à des praticiens -nos lointains ancêtres restaurateurs - choisis pour leur savoir-faire en ce domaine.

\section{Peindre}

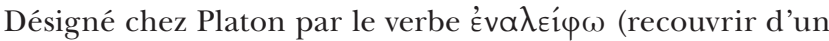
enduit) ${ }^{3}$, auquel font écho, dans les inscriptions hellénistiques de Délos, les nombreuses mentions d'aloïphè (application d'un badigeon, d'un enduit ou d'un vernis de poix) ${ }^{4}$, le travail de finition de la surface du marbre est désormais mieux connu grâce aux nombreux dossiers de sculpture hellénistique récemment étudiés. Le lecteur intéressé par les techniques de la polychromie sculpturale pourra donc se référer à d'autres publications ${ }^{5}$. Le propos se limitera ici au rappel de deux points.

Tout d'abord, on a pu mettre en évidence l'existence de pratiques communes dans la technè picturale du monde hellénistique. Parmi ses caractéristiques figurent l'usage du blanc de plomb (en grec psimithion) comme préparation et/ou comme matière picturale, pure ou mélangée avec d'autres teintes, la science du broyage des pigments et des mélanges, la finesse d'application des couches renvoyant à la notion grecque d'akrivéia (précision, justesse). L'inconnue profonde qui a longtemps entouré la nature des liants commence à céder. Ainsi, une gomme (acacia ?) a été détectée sur le trône peint et doré de la tombe d'Eurydice, de la tempera à l'œuf dans d'autres cas ${ }^{6}$. La technique à l'encaustique, bien attestée par les écrits antiques, reste pour l'heure étonnamment absente du paysage, alors que la recherche moderne s'est longtemps focalisée sur elle et a, en maintes occasions, fantasmé sa présence. Il est vrai que la seule image montrant le travail de peinture d'une statue, dans l'iconographie grecque, illustre justement le procédé de la cire à chaud. Sur le cratère apulien de New York (fig. 2), n'est-ce pas en effet une spatule que tient le praticien, tandis que son aide fait chauffer d'autres instruments semblables sur un brasero? La scène est donc généralement interprétée comme la représentation d'un peintre à l'encaustique procédant à la finition colorée d'une statue d'Héraclès en marbre, déjà installée sur sa base dans un temple; la naissance de l'image mimétique (la statue peinte) s'opère sous les yeux étonnés du héros lui-même, présent à la droite de l'artiste/artisan ${ }^{7}$.

Le second point concerne l'importance de cette parure colorée dans le sens conféré à l'objet, ainsi que dans la vision produite -une vision de chatoiement et d'éclat, où peinture et sculpture jouaient en complémentarité selon des jeux d'ombres et de lumières, des effets d'accentuation ou de substitution du modelé plastique par la peinture qu'on ne soupçonnait guère jusque-là. Les marbres que nous voyons blancs étaient richement peints et/ou dorés, carnations comprises; les draperies dont nous admirons l'ordonnance formelle avaient un fond coloré (pouvant être différencié entre avers et revers), orné

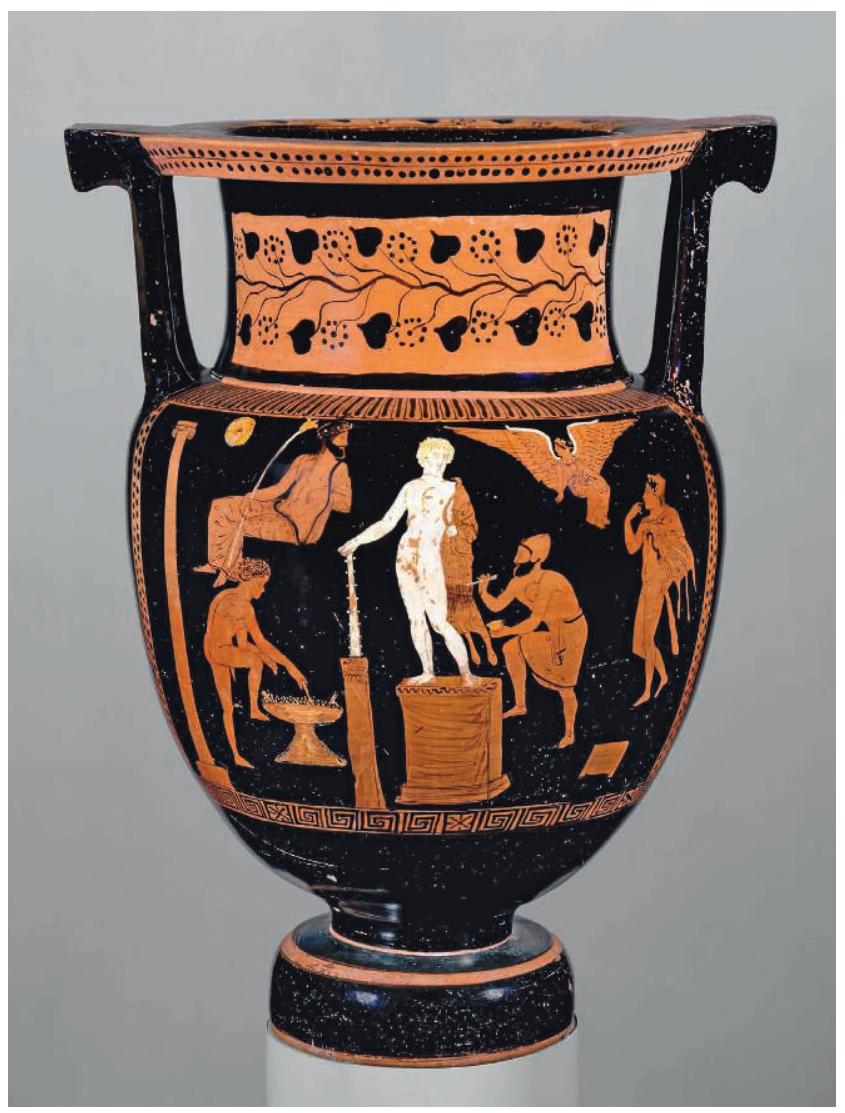

Fig. 2. Cratère apulien à colonnettes, attribué au Groupe de Boston 00.348, vers 360 av. J.-C. :

un artiste peignant à l'encaustique une statue d'Héraclès, New York, Metropolitan Museum of Art, inv. 50.11.4.

(C) The Metropolitan Museum of Art, Dist. RMN-

Grand Palais/image of the MMA. 
Fig. 3 a. Statuette fragmentaire d'Aphrodite, marbre blanc peint, Musée de (C) EFA/Philippe Collet. Délos, inv. A 5222
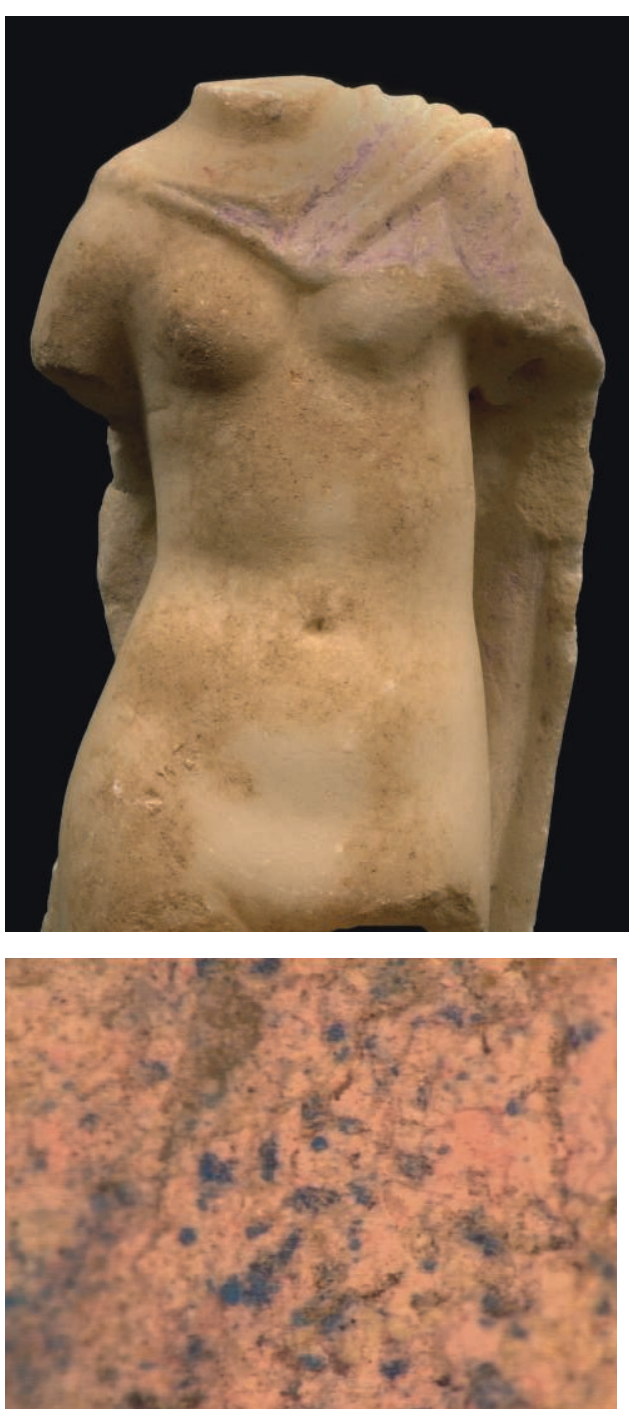

de bandes multicolores, de galons dorés, de franges et d'autres motifs. La sculpture peinte transcrivait ainsi le luxe et le raffinement des étoffes dont se parait la riche clientèle des villes prospères comme Délos. Évocatrice de la pourpre, la teinte mauve est l'un des tons que l'on retrouve le plus fréquemment dans la production des ateliers de l'île comme l'illustre, parmi bien d'autres, une petite Aphrodite au corps nu savamment mis en valeur sur le fond d'un manteau porté en cape (fig. 3 a-b). Le peintre n'a pas eu recours ici au précieux colorant extrait du murex; il a employé la formule de substitution «bon marché » consistant à mélanger du rose de garance et du bleu égyptien (fig. 4). Du plomb, détecté en $\mathrm{XRF}$, est présent également dans la matière picturale; il s'agit sans doute de blanc de plomb.

\section{Nettoyer, effacer}

Un enduit de surface pouvait être aisément effacé d'une statue, comme l'évoque un fragment d'une tragédie d'Euri-

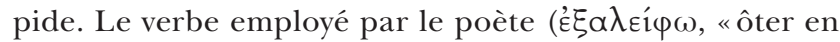
frottant») fait écho au terme utilisé par Platon, cité plus haut ${ }^{8}$.

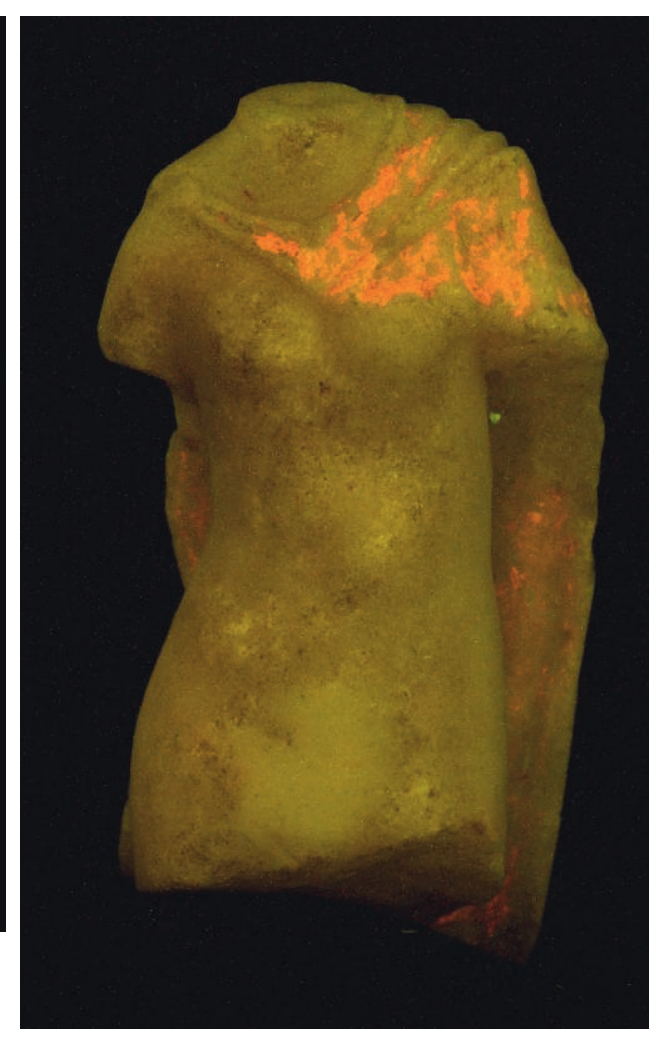

Fig. 3 b. Idem sous $\mathrm{UV}$ : la vive

fluorescence rouge de la peinture du manteau est caractéristique de la garance. (c) EFA/Philippe Collet.

Fig. 4. Idem, la peinture mauve est formée d'un mélange de bleu égyptien et de rose de garance (vidéomicroscopie, $x$ 110). (C) C2RMF-EFA Brigitte BourgeoisPhilippe Jockey.

Comme Evridiki Leka l'a rappelé, la thérapéia des statues comportait fréquemment des travaux de nettoyage. En effet, laisser la noire salissure ternir l'épiphanie divine était un signe de coupable négligence des hommes envers les dieux. Une œuvre de prestige comme le colosse chryséléphantin du Zeus d'Olympie, façonné par Phidias, avait son personnel de nettoyage attitré ${ }^{9}$. Peut-être en allait-il de même pour l'Aphrodite de Cnide? Le chef-d'œuvre sculpté par Praxitèle peu avant 360 av. J.-C., l'un de ces marbres achevés sans doute par le pinceau de Nicias, demeura pendant des siècles dans l'un des temples de la cité, doté d'un dispositif spécial de double porte permettant de contempler la statue de face et de dos ${ }^{10}$. L'afflux de pèlerins et d'admirateurs, accourus à Cnide pour voir l'œuvre réputée supérieure à tout autre sur terre, n'était sans doute pas anodin en termes de conservation, sans même parler des gestes de folie provoqués par l'irrésistible sensualité de la figure. Tel jeune homme devait ainsi se cacher dans le temple durant la nuit et, sacrilège, laisser au matin sur la cuisse de la déesse une tache honteuse ${ }^{11}$. Les auteurs chrétiens, dont Prudence, auront beau jeu par la suite de dénoncer ces «marbres souillés par d'infectes éclaboussures» qu'il convient de laver, en une sorte de baptême transformant 
l'idole corrompue en une pure œuvre d'art ${ }^{12}$. Tel Arnobe, cité en exergue, ils ridiculiseront ces dieux noircis, écaillés, vermoulus, couverts de fientes et de toiles d'araignées, à la merci de la décrépitude sitôt la thérapie humaine interrompue $^{13}$.

Comment juger des effets des nettoyages antiques? On se souvient que les méthodes employées semblent avoir été variées, au dire des quelques sources textuelles dont on dispose. Le lavage à l'éponge avec une solution saline (eau additionnée de natron) est bien attesté à Délos à l'époque hellénistique. Des siècles plus tard, Arnobe raillera ces dieux païens qui se salissent ("sordescunt et dei») et les ablutions à la lessive de cendres qu'on leur prodigue ${ }^{14}$. «Râcler» ( $\left.\xi u ́ \omega\right)$ fait aussi partie des pratiques - ainsi d'Ôphélion à Délos restaurant les trois statues du Pythion, avant de repeindre à l'encaustique et dorer ${ }^{15}$. En écho lointain, la voix de Minucius Félix qu'évoque Béatrice Caseau se moque de ces vaines idoles que les païens sont soucieux de sans cesse «essuyer, nettoyer, râcler ${ }^{16}{ }$. S'il demeure difficile de reconnaître l'impact de tels procédés sur les œuvres parvenues jusqu'à nous, l'entreprise s'avère parfois concluante. Les traces d'un profond raclage repérées sur le Torse de Milet au Louvre relèvent sans doute d'un nettoyage effectué à la ripe sur un original du style sévère; celui-ci a peut-être connu un premier enfouissement avant d'être restauré et remis en honneur dans le théâtre de Milet au $\mathrm{II}^{\mathrm{e}}$ siècle ap. J.-C. ${ }^{17}$. Et les remaniements de surface antiques décelés sur le portrait dit de Bérénice II à Mariemont ont comporté aussi un grattage partiel (voir plus bas).

Que l'action ait été chimique ou mécanique, ces nettoyages devaient affecter, voire assez souvent effacer des revêtements fragiles; d'où les nécessaires reprises de peinture, de dorure et de ganôsis que mentionnent fréquemment les comptes des sanctuaires.

\section{Repeindre: l'Asclépios de Dresde}

Qu'en est-il maintenant de l'évidence matérielle de repeints antiques dans le domaine de la sculpture grecque d'époque hellénistique? Contrairement à ce qu'on pourrait croire, la primeur de la découverte ne revient pas à notre époque bardée d'équipements scientifiques. La voie avait été ouverte par l'archéologue Georg Treu, grand connaisseur et ardent défenseur de la polychromie sculpturale, à la fin du XIX siècle $^{18}$.

En 1891, Treu, alors directeur de la collection de sculptures de l'Albertinum à Dresde, faisait acheter pour le musée un torse de statuette acéphale, drapé, en marbre blanc, représentant vraisemblablement Asclépios et provenant de Cos ${ }^{19}$. Mû par son habituel souci d'objectivité scientifique, il commanda au peintre allemand Ludwig Otto un relevé précis à l'aquarelle des traces de polychromie. En effet, non seulement la peinture qui couvrait le manteau avait largement survécu à l'enfouissement, mais, de plus, le regard exercé du savant y avait reconnu une stratigraphie plus complexe que d'ordinaire : il apparaît clairement, disait-il, «que le manteau porte deux couches de peinture superposées, l'une, inférieure, d'un rouge brique clair, l'autre supérieure d'un rose tendre ${ }^{20}$ ». La figure, concluait-il, avait donc été repeinte à une époque ultérieure.

Tombée quelque peu dans l'oubli par la suite ${ }^{21}$, cette assertion vient d'être confirmée par l'étude scientifique que Paolo Liverani et Ulderico Santamaria ont récemment menée ${ }^{22}$. Sous les concrétions archéologiques qui scellent le dernier état antique de surface, des restes de rose posés sur du rouge sont bien visibles. La coupe stratigraphique d'un échantillon confirme qu'une couche picturale rouge a été recouverte par une épaisse préparation blanche; sur cette dernière, le rose a été posé (fig. 5) - un rose vraisemblablement formé de garance à en juger par la vive fluorescence émise sous UV. La présence de la préparation, qui masque le rouge et l'efface de la vue, prouve qu'il s'agit bien d'une reprise diachronique, et non d'un procédé pictural ayant recouru à la superposition de couches différemment colorées ${ }^{23}$. Dans le domaine de la terre cuite, en revanche, où nous avons vu plusieurs cas d'application de rose de garance directement sur une sous-couche rouge, parmi la production hellénistique tardive à Délos et à Myrina, il s'agit bien d’un procédé pictural visant à intensifier et à faire vibrer la couleur du pigment laqué 24 .

Le repeint de la statuette de Dresde (dont on ne peut préciser la date) témoigne ainsi d'une inflexion du goût ayant privilégié le rose éclatant cher à l'époque hellénistique à un ton rouge plus traditionnel. L'évolution chromatique n'est pas sans rappeler ce que nous avons détecté par ailleurs sur des statuettes en terre cuite repeintes dans l'Antiquité, comme la Ménade attique, dite Dame Baillehache, ou bien un Apollon archer de Délos 25

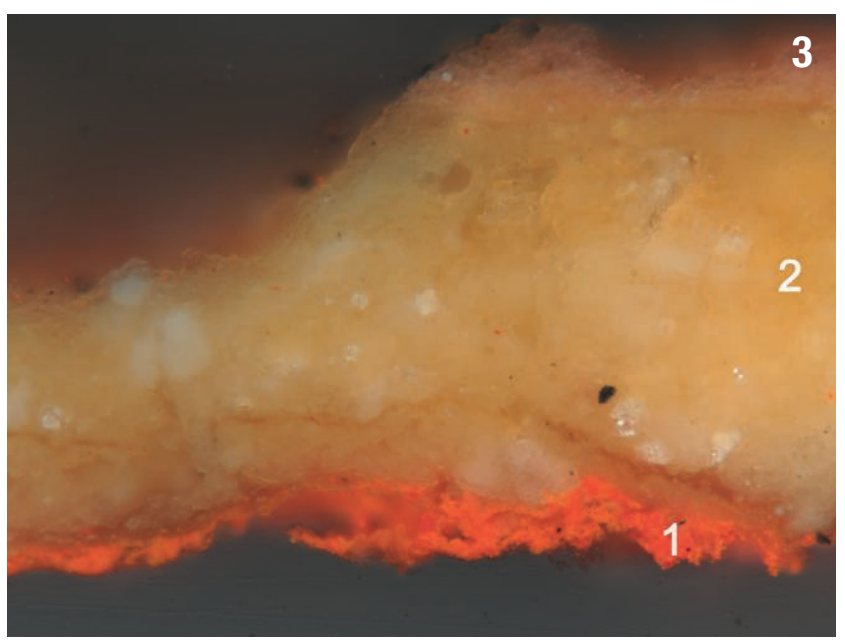

Fig. 5. Asclépios de Dresde, coupe stratigraphique du repeint antique. Couche 1 (peinture rouge): premier état; couches 2 (préparation blanche) et 3 (couche picturale rose) : second état.

(C) P. Liverani-U. Santamaria. 


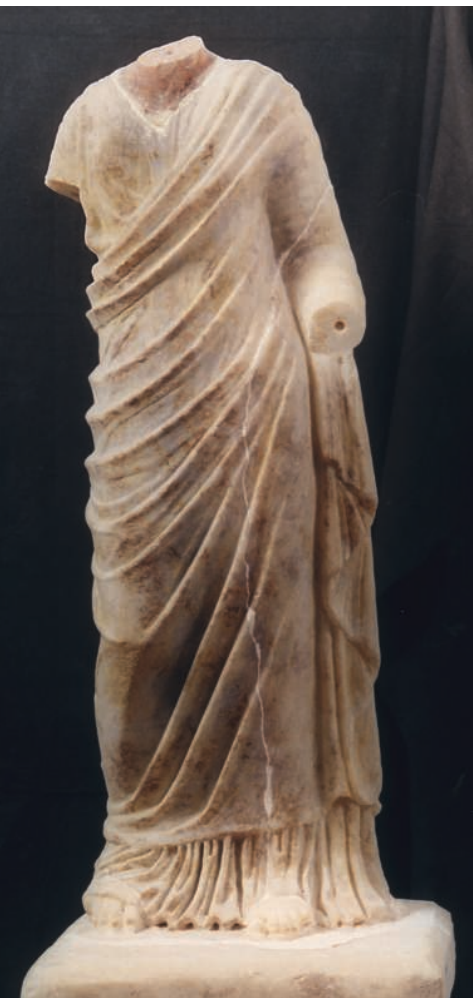

Fig. 6. Statuette féminine dorée, provenant de la Maison des Masques, $\mathrm{II}^{\mathrm{e}}$-début du I ${ }^{\mathrm{er}}$ s. av. J.-C. H. $55 \mathrm{~cm}$, marbre blanc et gris-bleu, avec peinture et dorure, Musée de Délos, inv. A 4134. Ensemble face. (C) EFA/Philippe Collet.

\section{Peindre puis dorer: la statuette de la Maison des Masques à Délos}

Dans le domaine délien justement, si riche d'enseignements, était-il possible de corroborer par des traces matérielles les nombreux témoignages épigraphiques mentionnant des remises en état d'effigies? Quoique difficile ${ }^{26}$, l'entreprise s'est révélée concluante, et une première attestation peut être versée au dossier, sur la base des résultats acquis par le programme de recherche conduit il y a quelques années par Philippe Jockey et moi-même ${ }^{27}$.

L'œuvre concernée est la statuette féminine dorée, trouvée en 1930 dans la Maison des Masques (fig. 6). La figure à l'identité rendue incertaine par la perte de la tête et des bras (Aphrodite drapée? autre divinité?) est représentée debout, vêtue d'une tunique longue et d'un grand manteau enroulé autour du corps ${ }^{28}$. Visibles à l'œil nu lors de la fouille, les restes de la dorure du vêtement n'avaient pas tardé à s'altérer rapidement à l'air libre comme Jules Chamonard, l'inventeur de l'œuvre, l'avait rapporté et comme le confirmait un peu plus tard Jean Marcadé, dans les études qu'il consacrait aux effigies dorées déliennes ${ }^{29}$. À l'heure actuelle, seule l'assiette jaune ferrugineuse qui portait la dorure (selon la technique dite «au bol») couvre encore largement la surface du marbre sous les concrétions d'enfouissement, tandis que l'observation des restes de feuille d'or nécessite l'appui de la microscopie (fig. 7). L'assiette est posée sur une couche irrégulière de préparation blanche dans laquelle l'analyse en fluorescence $\mathrm{X}$ portable a détecté un net signal du plomb ${ }^{30}$. Dans le cas présent, nous avions envisagé l'hypothèse que le blanc de plomb ait été choisi en raison de son pouvoir couvrant; en effet, la statuette relève de la technique mise en évidence par $\mathrm{Ph}$. Jockey, dite technique composite à pièces rapportées en marbres de différentes qualités (blanc pour les chairs, grisbleuté pour le corps drapé) ${ }^{31}$. De la sorte, la disparité chromatique du support était atténuée, voire abolie.

Cependant, il y a plus. Lors de l'examen en vidéo-microscopie, des restes de peinture rose posés sur une sous-couche blanche sont apparus, en divers endroits du drapé, sous les couches liées à la dorure (fig. 8). D’autre part, la photographie sous UV (fig. 9) a révélé des plages éparses de fluorescence rougeorangée, qui ne peuvent en rien se rapporter à la technique de dorure ici employée, mais qui semblent bien indiquer la présence d'un colorant tel que la garance dans la composition du rose. Ces deux indices, ténus mais indubitables, plaident en faveur d'une réfection antique de kosmèsis. D'abord peint

Fig. 7. Statuette féminine dorée, reste de dorure à la feuille sur le manteau (vidéo-microscopie, x 100). Couche 1 : marbre gris-bleu, couche 2: sous-couche blanche contenant du blanc de plomb, couche 3 : assiette jaune ferrugineuse, couche 4 : reste de feuille d'or, couche 5: concrétions d'enfouissement. (C) C2RMF-EFA/ Brigitte Bourgeois-Philippe Jockey.
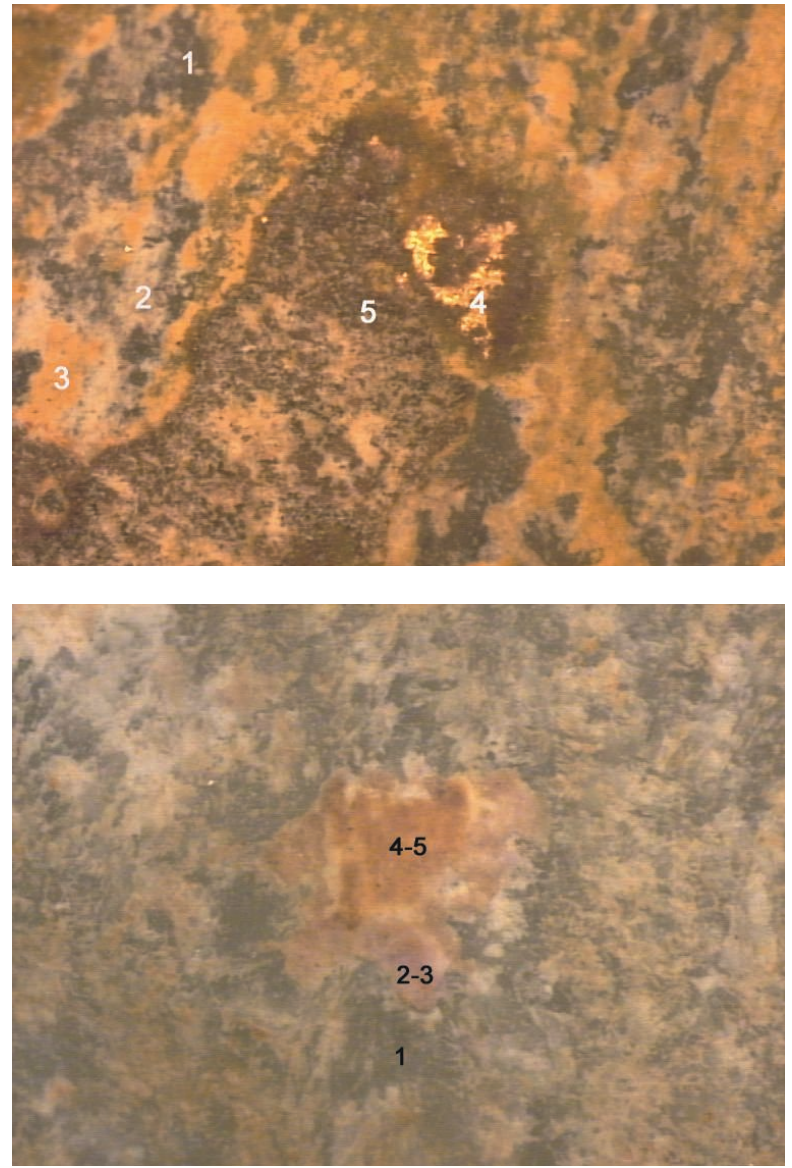

Fig. 8. Idem, vestige des deux états de polychromie antique (vidéomicroscopie, x 140). Couche 1 : marbre, couches 2-3: premier état (peinture rose sur préparation blanche), couches 4-5: deuxième état (assiette jaune de la dorure sur nouvelle préparation blanche). (C) C2RMF-EFA/Brigitte Bourgeois-Philippe Jockey. 


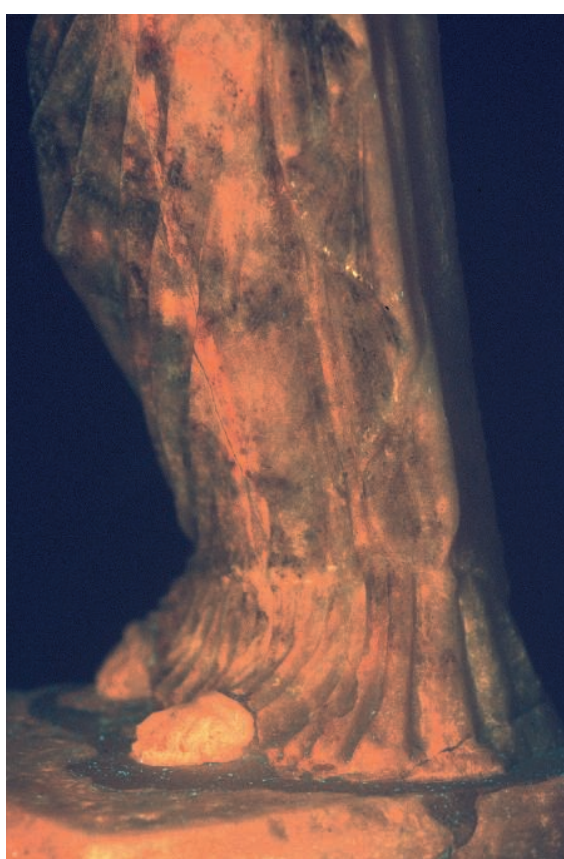

Fig. 9. Statuette féminine dorée sous UV, montrant la fluorescence rouge caractéristique de la garance. (C) EFA/Philippe Collet.

en rose, le manteau aura été doré dans un second temps. À en juger par les vestiges réduits de rose sous dorure, il semble bien que l'artisan antique ait éliminé (par lavage ou grattage?) le décor initial, avant de ré-enduire la surface d'une couche de préparation sur laquelle il a procédé à la dorure.

Dans quel contexte l'opération a-t-elle été menée et où? Nous l'ignorons. Était-ce sur place ou dans un atelier de peintre tel celui récemment découvert par P. Hatzidakis dans l'Allée des Lions ${ }^{32}$ ? S'agit-il d'une pieuse remise en état, d'un changement de fonction de l'œuvre ou bien de la manifestation d'un goût de luxe chez un propriétaire "nouveau riche ${ }^{33}$ ? Parlant de l'usage du marbre doré à Délos, Jean Marcadé soulignait la rareté d'un tel traitement de surface à l'époque hellénistique; en dehors des sanctuaires, écrivait-il, «dans les quartiers périphériques, on n'imagine guère que certaines "statuettes de laraire" portant un revêtement d'or ${ }^{34}$ ". Même si, au vu des découvertes engrangées depuis lors, le jugement sur la faveur de l'or à Délos doit être nuancé, l'argument n'a pas perdu de sa force pour autant et incite à attribuer à la statuette de la Maison des Masques une fonction cultuelle plutôt qu'une simple valeur décorative ${ }^{35}$.

\section{(Re)peindre, dorer, cirer. Un cas complexe de thérapéia: le portrait de Bérénice II à Mariemont}

\section{Un portrait royal de l'Égypte ptolémaïque peint et doré}

Le Musée royal de Mariemont, en Belgique, conserve une tête féminine en marbre blanc (fig. 1), proche de la grandeur nature, issue de la collection de Raoul Warocqué qui l'avait achetée en vente à l'Hôtel-Drouot en $1905^{36}$. Au cours du $\mathrm{XX}^{\mathrm{e}}$ siècle, l'œuvre acquit une célébrité particulière en raison de sa grande qualité sculpturale, de la présence remarquable de polychromie, associant peinture et dorure, ainsi que de l'identité de la figure. En effet, la tête a été rapprochée de l'iconographie des souverains lagides et identifiée, selon la plupart des spécialistes, comme un portrait de Bérénice II, l'héritière du royaume de Cyrénaïque qui devint, par son mariage avec Ptolémée III Evergète, reine d'Égypte de 247/246 à 221 avant J.-C. ${ }^{37}$. On sait également, depuis l'étude de Roland Tefnin parue en $1969^{38}$, que la tête provient d'Égypte, plus précisément du site d'Hermoupolis Magna, comme l'atteste un ancien témoignage publié dans la littérature archéologique des années 1900: alors actif sur le terrain, Otto Rubensohn avait rapporté la trouvaille, survenue durant l'hiver 1901 entre les mains de propriétaires locaux, de vestiges d'un temple égyptien, de papyri et de nombreuses sculptures de marbre, dont cette tête. Sans en donner de description précise, il mentionnait bien la présence de compléments en stuc et de nombreux restes de peinture sur la chevelure et le visage $^{39}$.

Cette polychromie est restée bien présente, et l'intensité expressive du portrait ne manque pas d'impressionner dès le premier regard. Le rendu pictural des traits du visage et des courtes mèches noires qui encadrent celui-ci en constitue l'élément le plus visible. Le traitement des yeux est particulièrement détaillé: les longs sourcils sont rendus par une ligne noire (doublée d'un trait rouge pour l'œil droit), barrée de petits traits perpendiculaires détaillant les poils. Le cercle de l'iris et les cils sont peints en noir tandis que des traits rouges et noirs soulignent les bords des paupières. Quelques rehauts de rouge sur la bouche et le sillon naso-labial complètent cette polychromie austère, faisant appel à une gamme chromatique limitée au noir, au brun et au rouge. Il est plus difficile en revanche de visualiser la dorure; celle-ci ne subsiste plus qu'à l'état de traces ténues sur la narine gauche, aux tempes et autour de l'œil gauche. Enfin, il est frappant de constater combien ce beau visage paraît marqué et comme «sali » par la présence d'un voile jaunâtre, couvrant notamment la joue droite, ainsi que par des résidus d'un badigeon brun-rouge, présent sur le bas de cette même joue et dans le cou.

La présence simultanée de vestiges peints et dorés a donné lieu à diverses interprétations dans le passé, sur la base d'observations strictement visuelles. R. Tefnin y a vu un seul et même état, associant «l'éclat d'or du visage» d'une reine, divinisée de son vivant, à l'animation de rehauts peints ${ }^{40}$. D'autres auteurs, comme Helmut Kyrieleis, y ont vu la preuve d'un remaniement antique, en envisageant un portrait peint puis doré ${ }^{41}$.

Soucieuse d'apporter au débat des éléments scientifiques plus précis, Annie Verbanck-Piérard, responsable de l'œuvre au Musée royal de Mariemont, m'a demandé d'approfondir l'étude de la polychromie du portrait. Les résultats qui vont suivre ont été établis sur la base d'examens en vidéo-microscopie et en microscopie 3D, de photographies UV, et de premières mesures d'analyse d'abord non invasive puis sur prélèvements ${ }^{42}$. 


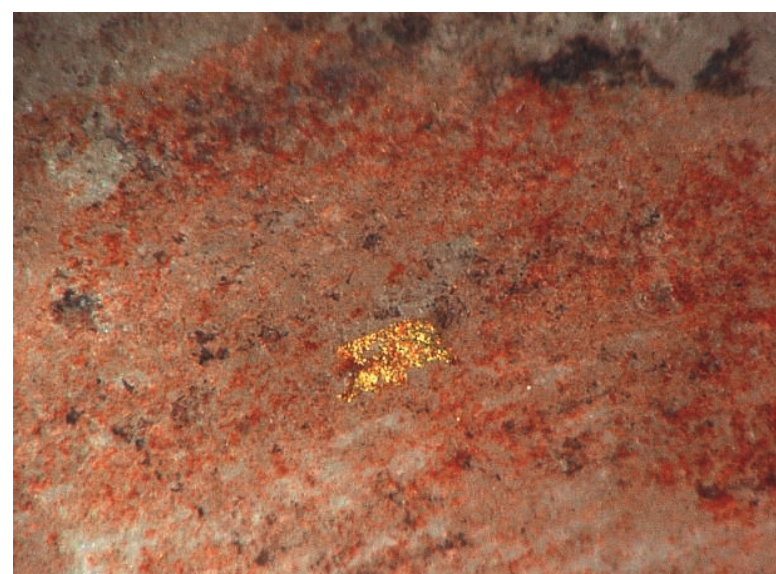

Fig. 10. Portrait de Bérénice II, trait de peinture rouge dans la partie inférieure du sourcil droit: reste de feuille d'or sous le rouge (vidéo-microscopie, x 100). (c) C2RMF/Brigitte Bourgeois.

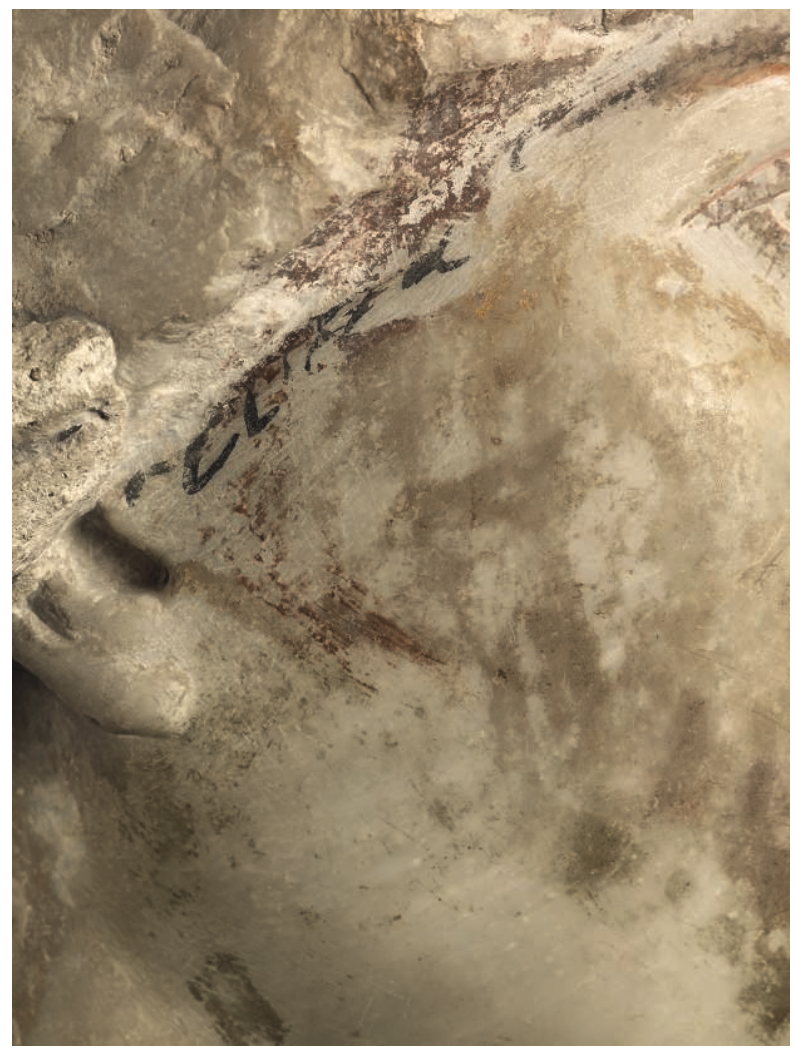

Fig. 12. Idem, tempe, joue et oreille droite. (C) Musée royal de Mariemont/Anne Chauvet.

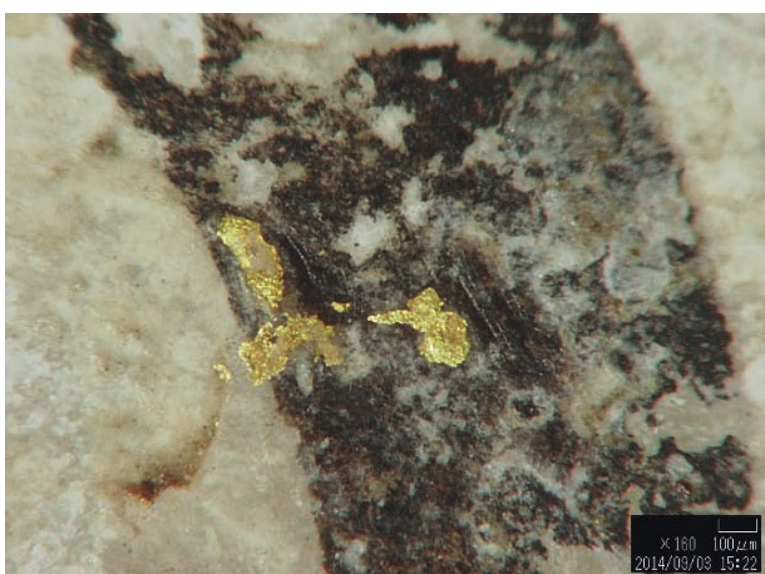

Fig. 11. Idem, sur la tempe gauche, mèche noire (vidéo-microscopie, x 160). Reste de feuille d'or appliquée sur la peinture noire.

(c) C2RMF/Brigitte Bourgeois.

\section{Signes de repeints antiques}

Il apparaît désormais que loin d'être cohérente, la finition colorée s'avère déconcertante et plus complexe que ce qu'envisageaient les scénarios précédents. Les hypothèses d'un visage tout doré avec des rehauts peints sur la dorure (Tefnin) ou d'un visage peint puis doré (Kyrieleis) devraient montrer des strates de peinture et de dorure superposées. Or tel n'est pas le cas. Très rares sont en fait les vestiges (de taille microscopique) qui associent les deux types de traitements, de manière d'ailleurs contradictoire: tantôt un vestige de feuille d'or transparaît sous une couche peinte (par exemple dans le trait rouge sous le sourcil droit) (fig. 10), tantôt il apparaît surune couche peinte (par exemple sur l'une des mèches peintes en noir sur la tempe gauche) (fig. 11).

On notera d'autre part que l'état du support en marbre est lui-même hétérogène (fig. 12). Il juxtapose des zones parfaitement polies, comme le bas de la joue droite, par exemple, à d'autres plages visiblement grattées, au vu des fines stries plus ou moins parallèles qui rayent le marbre. C'est le cas de la tempe droite, en lisière de la chevelure. Par-dessus ces stries ont été peintes de petites mèches noires. Les irrégularités, comme la raideur du tracé en crochet de certaines d'entre elles, contrastent avec le dessin plus fluide et régulier d'autres mèches, situées plus haut vers le front ou sur la tempe gauche. Enfin, des restes d'une couche de peinture brun-rouge passent surces mèches en crochet de la tempe droite (fig. 13). Ces dernières ont donc été masquées en totalité ou en partie, dans un temps ultérieur à leur application, par cette sorte de badigeon rougeâtre qui semble également avoir recouvert le bas du visage et certaines plages du cou, en masquant là encore un motif préexistant de petites mèches noires.

À regrouper ces indices, il semble que seule l'hypothèse de remaniements, ayant procédé par grattage et/ou par recouvrement partiel des états antérieurs via des motifs « de seconde main ", puisse rendre compte de toutes ces anomalies.

Faut-il soupçonner dans tout ceci des repeints modernes? Outre le témoignage de Rubensohn, qui a vu l'œuvre au sortir 


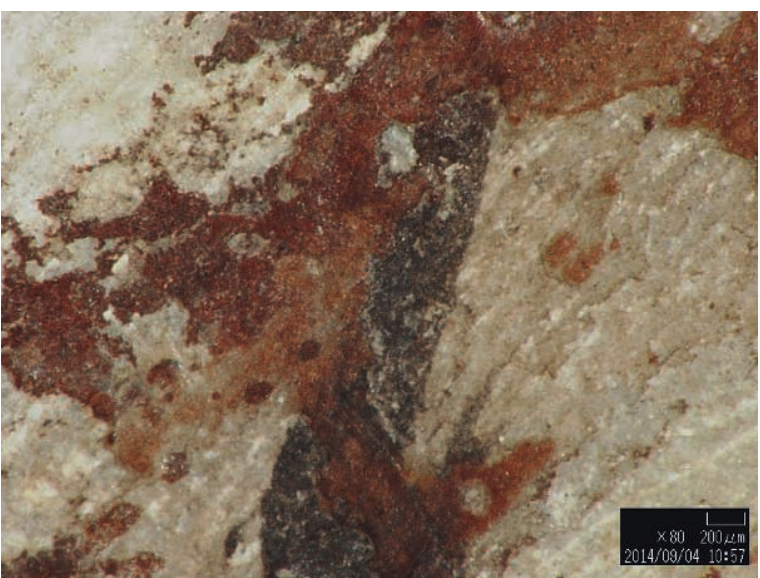

Fig. 13. Repeint en peinture brun-rouge sur la deuxième mèche noire peinte en crochet au-dessus de l'oreille droite (vidéo-microscopie, x 80). (c) C2RMF/Brigitte Bourgeois.

de terre et atteste la présence de peinture antique, on fera observer que l'état d'altération des vestiges, portant fréquemment des dépôts d'enfouissement, témoigne bien plutôt du caractère archéologique de la surface. L'aspect général de la tête ne plaide pas non plus en faveur d'une cosmétique moderne: marqué par des discordances chromatiques d'une plage à l'autre, notamment du fait de la présence du voile jaunâtre sur la joue droite, il frappe de prime abord par son caractère désaccordé et somme toute peu flatteur. Une main moderne de restaurateur ou de faussaire cherchant à valoriser la tête sur le marché de l'art eût procédé autrement, en enjolivant ou en harmonisant sous des jutages un état archéologique disparate. L'état du portrait a d'ailleurs peu changé au cours du Xx ${ }^{\mathrm{e}}$ siècle, comme l'attestent les archives photographiques de Mariemont ${ }^{43}$ et les témoignages oraux; le musée a surtout veillé, avec une extrême prudence, à conserver la polychromie sans jamais chercher à purifier le visage de ses taches disgracieuses par un nettoyage poussé. Ce faisant, il a préservé un rare témoignage d'ancienne thérapéia: un vernis de cire.

\section{Un glaçage de surface à la cire d'abeille: l'évidence en faveur d'une opération de ganôsis}

Durant l'examen microscopique, il m'était apparu qu'outre la peinture et la dorure, un revêtement d'une autre nature subsistait à certains endroits du visage, sous forme d'une pellicule luisante et craquelée, de couleur jaunâtre, parfois plus grise, portant elle aussi des sédiments d'enfouissement. Les vestiges étaient bien nets par exemple sur le lobe de l'oreille droite (fig. 14) ou dans la conque de l'oreille gauche (fig. 15). À plus fort grossissement, la substance paraissait translucide; émettant une fluorescence blanche marquée sous UV, elle évoquait une matière organique telle que la cire.

L'hypothèse a été soumise à analyse et pleinement validée grâce à l'étude de deux micro-échantillons, prélevés l'un dans l'oreille gauche et l'autre dans le haut du cou à gauche

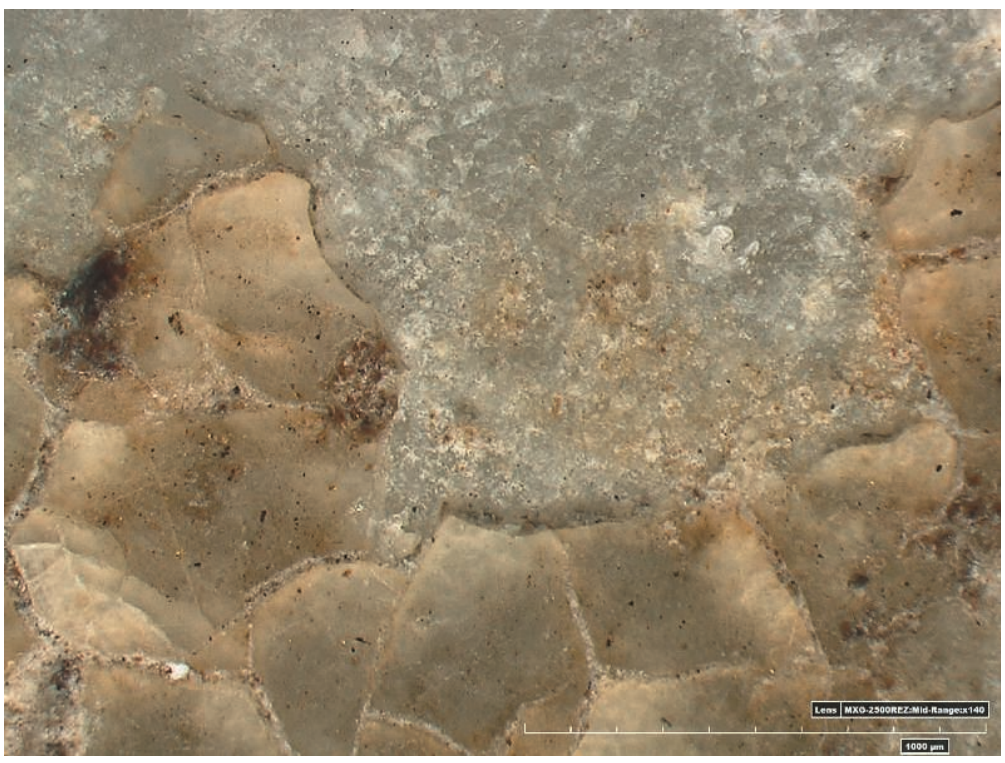

Fig. 14. Sur l'oreille droite, couche craquelée de surface (cire d'abeille) sous des dépôts d'enfouissement (microscope 3D x 140). (C) C2RMF/Brigitte Bourgeois, Dominique Robcis.

Fig. 15. Dans l'oreille gauche, couche de cire d'abeille sous les dépôts d'enfouissement et zone de prélèvement de l'échantillon 1 (voir article de Balcar, Langlois, Vandenberghe dans le présent volume) (vidéo-microscopie, x 20). (c) C2RMF/Brigitte Bourgeois.

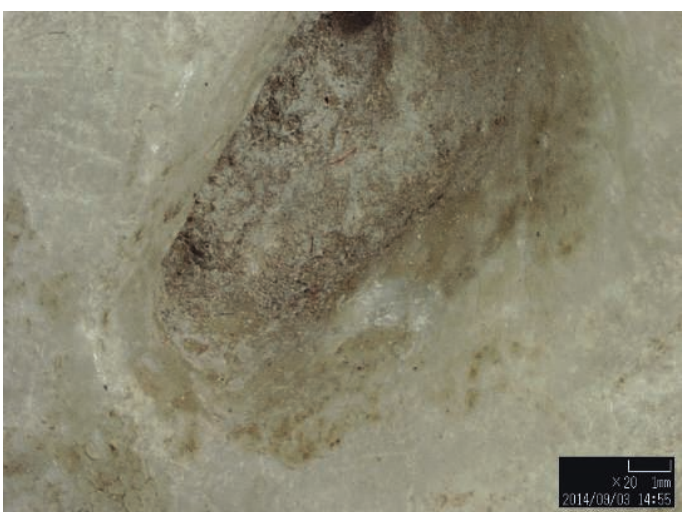




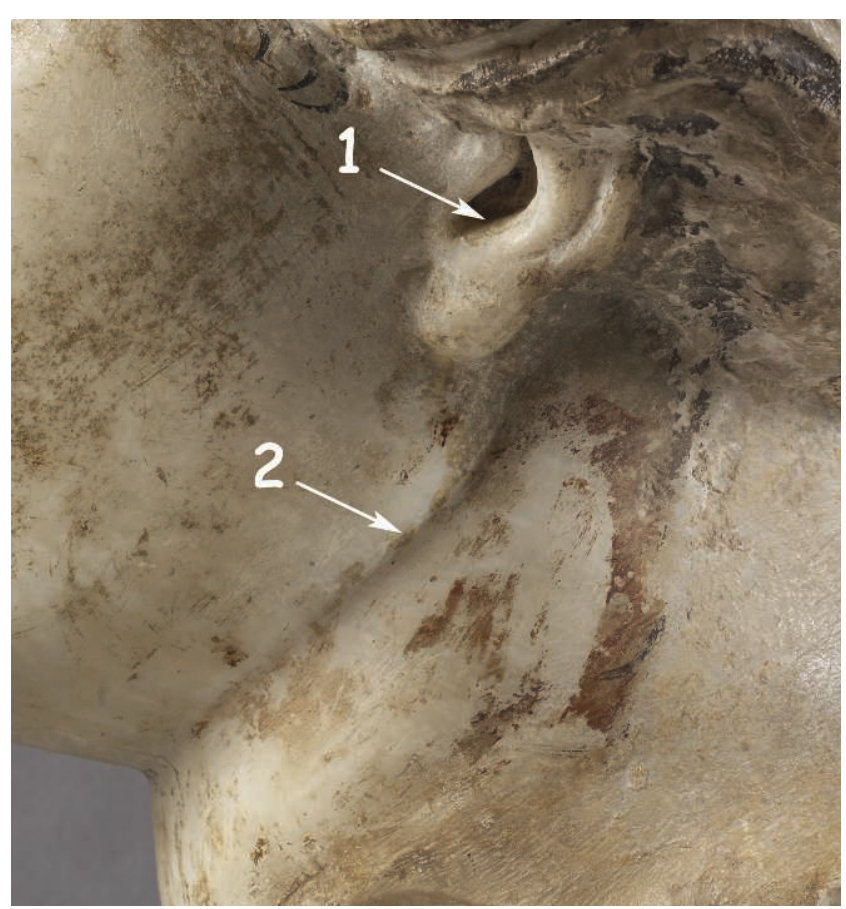

(fig. 16) ${ }^{44}$. Dans les deux cas, la composition correspond à une cire d'abeille pure, sans adjonction d'huile, ni de résine ni de pigment. Ce dernier point est particulièrement important: il prouve qu'il s'agit bien d'une enduction de surface du type d'un vernis, et non d'une technique de peinture à l'encaustique.

Étant donné la priorité accordée à l'identification du matériau, aucune autre mesure (datation par exemple) n'a pu être effectuée. Ne pourrait-il donc s'agir d'un traitement à la cire $\mathrm{du} \mathrm{XX}^{\mathrm{e}}$ siècle ? À cette question légitime, on peut répondre par une série d'indices indiquant que le procédé
Fig. 16. Vue du profil

gauche avec l'emplacement

des deux prélèvements de

cire. (c) C2RMF/Brigitte

Bourgeois.

relève bien d'une application intentionnelle, et est antérieur à l'enfouissement: $1^{\circ}$ ) Vient d'abord la morphologie. Il s'agit bien d'une couche, appliquée de manière régulière, dont on a pu mesurer l'épaisseur (80-100 microns) en microscopie 3D (fig. 17) et en microscopie électronique à balayage ; l'hypothèse d'une contamination fortuite dans le sol ou après l'exhumation est donc exclue. $2^{\circ}$ ) La stratigraphie montre bien la présence de dépôts d'enfouissement sur la cire et dans les craquelures. $3^{\circ}$ ) Le faciès d'altération est marqué : la cire a été affectée aussi bien par l'altération mécanique (réseau de craquelures prononcé) que par l'altération chimique; l'analyse par chromatographie en phase gazeuse a mis en évidence la dégradation survenue, par perte des acides gras et des alcanes, analogue à celle de la cire étudiée sur les portraits du Fayoum.

S'ajoutent à ces éléments d'autres considérations relatives aux enseignements de l'histoire de la restauration des marbres antiques. La pureté de la substance (cire d'abeille sans paraffine ni résine ni adjuvant) ne correspond guère à ce qu'on connaît des produits de restauration d'époque moderne ${ }^{45}$. La cire n'est conservée qu'à certains endroits, par exemple, non pas sur l'ensemble de l'oreille gauche, mais seulement dans le creux et en partie sur le bord. Or la pratique de cirer les marbres antiques, documentée dans le cadre de restaurations historiques en Italie et en France, s'appliquait à l'intégralité de la surface: l'exemple d'un grand marbre au long passé de restauration $\left(\mathrm{XVII}^{\mathrm{e}}\right.$-XIX ${ }^{\mathrm{e}}$ siècle) comme le Gladiateur Borghèse peut en témoigner ${ }^{46}$.

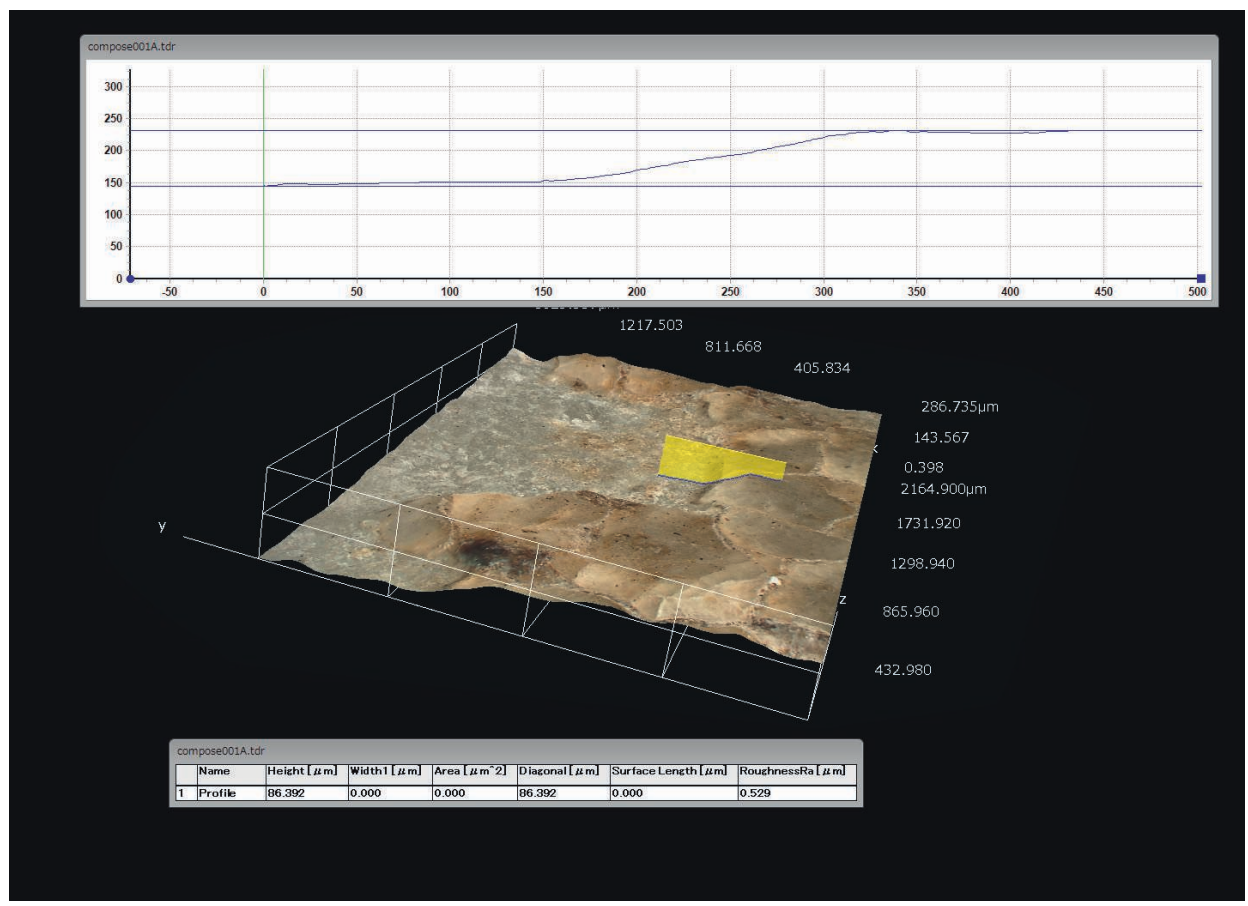

Bourgeois,

Dominique Robcis. 
La tête de Bérénice représente donc selon toute vraisemblance l'un des très rares témoignages de sculpture grecque ayant conservé trace d'un traitement du type de la ganôsis ${ }^{47}$. Aucun ton de carnation colorée n'est ici associé au glaçage à la cire. Des conditions de conservation particulière pourraient expliquer que ce dernier ait en partie survécu sur le portrait royal de Mariemont, surtout si l'effigie à laquelle il appartenait était placée dans un temple. La question rejoint là un débat ouvert: la statue, représentant la reine grandeur nature, étaitelle l'une des statues de culte (agalmata) que mentionne une dédicace en grec sur l'architrave d'un temple fouillé au cours du $\mathrm{XX}^{\mathrm{e}}$ siècle à Hermoupolis? D'après cette inscription, le bâtiment était consacré au culte des "dieux Evergètes" (Ptolémée III et Bérénice II divinisés), ainsi qu'au couple de souverains qui les avaient précédés, Ptolémée II et Arsinoé II «dieux Philadelphes» ${ }^{48}$. Ou bien n'aurait-elle pas été consacrée dans le temple égyptien (et non grec) que mentionnait Rubensohn, comme le rappelle François Queyrel ${ }^{49}$ ? Quoi qu'il en soit, la présence de cire fournit sans doute un argument supplémentaire en faveur d'une fonction cultuelle, si l'on pense que la ganôsis faisait partie des soins attentifs rendus par des artisans spécialisés dans la «remise en bel ordre» des effigies sacrées.

\section{Conclusion}

Le cas de la statuette délienne peut être utilement rapproché d'un point souligné par Véronique Chankowski dans son étude sur l'économie de la restauration ${ }^{50}$. On ignore souvent, écrit-elle, si les travaux de dorure mentionnés dans les comptes concernent «une remise en état ou une première dorure, réalisée longtemps après l'installation de la statue de culte». L'effigie féminine de la Maison des Masques, peinte puis dorée (sans qu'on puisse préciser quel laps de temps a pu séparer ces deux états) vient fournir une première attestation, dans l'île sainte, de la volonté de conférer une nouvelle apparence, encore plus éclatante et précieuse, à une image pieuse en contexte domestique.

Dans le cas de la tête de Mariemont, l'hétérogénéité des états de surface, marqués notamment par d'anciens grattages et repeints, brouille la lecture d'une ouvre palimpseste et rend plus difficile l'établissement d'une chronologie des remaniements. Ce qui est pris pour un «original antique» est bien plutôt la somme de modifications apportées, peutêtre sur une longue durée, à la création du $\mathrm{III}^{\mathrm{e}}$ siècle av. J.-C., et la ganôsis pourrait elle-même dater d'une de ces reprises. La poursuite programmée de l'étude apportera sans doute des éclaircissements sur la relation entre cire, peinture et dorure. Quoi qu'il en soit, la mise en évidence d'un vernis de cire d'abeille pure représente une nouvelle étape importante dans l'étude de la sculpture grecque. La ganôsis n'a longtemps été connue que par des sources textuelles. Voici que l'épiderme du marbre reprend corps dans ce qu'il a de plus impalpable (un voile incolore) - et de plus organique, longtemps sujet d'effroi pour bien des critiques d'art: le visqueux, le «gras» de la cire ${ }^{51}$. La pure forme de marbre blanc chantée par le poète ${ }^{52}$, éternelle et immuable dans sa dureté minérale, s'éloigne encore un peu plus de nous.
Notes

J'adresse mes plus vifs remerciements à Paolo Liverani et Ulderico Santamaria, pour m'avoir autorisée à reproduire un document de leur étude sur l'Asclépios de Dresde, à Annie Verbanck-Piérard pour son accueil à Mariemont et pour toutes les facilités accordées lors de l'étude du portrait de Bérénice, à Dominique Robcis pour son aide en microscopie 3D, et à Violaine Jeammet pour ses conseils et son attentive relecture.

1. Bloch, 2007, p. 53-54.

2. Appareillage utilisé à Délos: Keyence VH-5901 (résolution 470000 pixels, lentille zoom 25-175 fois). Pour l'étude du portrait de Bérénice II, appareil Hirox VCR-800 (résolution 2,1 millions de pixels, lentille zoom 20-160 fois) et microscope 3D Hirox KH-8700 avec lentille Revolver.

3. Platon, Politéia (La République), 420, c-d, et commentaires de Primavesi, 2008, p. $37-38$.

4. Hellmann, 1992, p. 37-40.

5. Von Graeve, Preusser, 1981 ; Bourgeois, Jockey, 2005, 2007, 2010 ; Kakoulli, 2009; outre les éditions successives du catalogue Bunte Götter depuis 2003, voir Brinkmann, Hollein, Primavesi, 2010, Blume, 2014, Brecoulaki, 2014, Bourgeois à paraître, etc.

6. Voir Brecoulaki à paraître.
7. Distinguo moderne sans équivalent en grec ancien. Sur le cratère, de Cesare, 1997, p. 103-105 et catalogue $\mathrm{n}^{\circ} 177$ p. 254 (avec bibliographie antérieure), Palagia, 2006, p. 261, Marconi, 2011 sur la «naissance de l'image».

8. Euripide, Hélène, 260-263, et commentaire Primavesi, 2008, p. 33-35.

9. Voir les articles de V. Pirenne-

Delforge et E. Leka.

10. Au témoignage de Lucien, Amours, 11, 13-14; Pasquier, 2007, p. 139-140.

11. Pline, Histoire naturelle, XXXVI, 20.

Et Lucien, Imag., 4, 6, 23.

12. Prudence, III, Contre Symmaque, 501 505, texte établi et trad. par M. Lavarenne,

Paris, Les Belles Lettres.

13. Arnobe, Adversus nationes, VI, 16, 6.

14. Id., VII, 32, 6 .

15. En 246 av. J.-C. Marcadé

1969, p. 100-101. Et Leka dans le présent volume.

16. Caseau dans le présent volume.

17. Braunstein, 1995

18. Sur lui, voir Elsner, Knoll, 1994.

19. Dresde, Albertinum, inv. Hm 215

Ht. $40 \mathrm{~cm}$. Treu, 1892. Étude récente de

Ch. Vorster (2009) qui le date des années

150-100 av. J.-C.

20. Treu, 1892, p. 159 (ma traduction)

21. L'aquarelle a figuré dans

l'exposition Bunte Götter à Munich, voir

Brinkmann, Wünsche, 2003, p. 17, puis dans l'édition italienne de l'exposition aux musées du Vatican (I colori del bianco, 2004, p. 21) afin de témoigner de l'intérêt de Treu pour la polychromie, mais sans mention du repeint antique.

22. Liverani, 2014, p. 10-11.

23. Par exemple sur une statue de Tychè (Musée de Délos, inv. A 4129), marbre provenant de la Maison des Cinq Statues: Bourgeois, Jockey, 2007, p. 166-167 et 180.

24. Voir l'article de B. Bourgeois et V. Jeammet dans ce volume.

25. Id. pour la Dame Baillehache. Sur l'Apollon archer (Musée de Délos, inv. A 394), Bourgeois, Jockey, 2007, p. 189-190.

26. Les fouilles de l'École française d'Athènes ont livré un abondant corpus de marbres, datant pour l'essentiel de la seconde moitié du $\mathrm{II}^{\mathrm{e}} \mathrm{s}$. et du début du $\mathrm{I}^{\mathrm{er}} \mathrm{s}$. av. J.-C., provenant le plus souvent de contextes domestiques et de lieux publics, parfois d'ateliers-boutiques; il ne correspond que rarement aux statues de culte et offrandes mentionnées par les inventaires et les comptes des sanctuaires. Il faut compter aussi avec l'altération d'un environnement fortement marqué par l'action de la mer, puis avec l'exposition à l'air libre et l'impact des conditions muséographiques.

27. C2RMF (B. Bourgeois)-EFA (Ph. Jockey), en collaboration avec Andréas Karydas (Démokritos, Athènes) et Hariclia 
Brecoulaki (Kera, Athènes). L'étude de la statuette a inclus photographie en

fluorescence UV, vidéo-microscopie, analyse élémentaire par spectrométrie de fluorescence X portable, analyse IRTF et chromatographie d'un échantillon (S. Sotiropoulou, Ormylia, et M.-P. Colombini, Pise).

28. Musée de Délos, inv. A 4134. Ht. $55 \mathrm{~cm}$. Datée vers la fin du II ${ }^{\mathrm{e}} \mathrm{s}$. av. J.-C.

29. Chamonard $B C H 54,1930$, p. 514 et 57, 1933, p. 157. Marcadé, 1969 et BCH 76, 1952, p. $98-135$.

30. Bourgeois, Jockey, 2005, p. 295-296.

31. Jockey, 1998.

32. École française d'Athènes, British School in Athens, Chronique des fouilles en ligne, notice $\mathrm{n}^{\circ} 847$ (www.chronique.efa.gr, consulté le 27 janvier 2014).

33. Brinkmann, dans Brinkmann, Wünsche, 2003, p. 178, interprète ainsi le goût pour le clinquant de l'or à Délos.

34. Marcadé, 1969, p. 114.

35. Bourgeois, Jockey, 2005, p. 315-316.

36 . Voir la notice de N. Massar dans Trésors de Mariemont. Collection Raoul Warocqué, 2007, p. 60-61. De 1902 à 1905, la tête a appartenu au collectionneur marchand P. Philip, établi au Caire. Une étude sur la polychromie du portrait, contenant une bibliographie plus détaillée, va bientôt être publiée (Bourgeois, à paraître).

37. F. Queyrel, 1984, p. 287, proposant pour sa part d'y voir un portrait d'Arsinoé III.

38. Tefnin, 1969

39. Rubensohn, Archäologischer Anzeiger, 1902, p. 47. Il reste encore une mèche en stuc au niveau de l'oreille droite.

40. Tefnin, 1969, p. 91

41. Kyrieleis, 1975, p. 181. Reuterswärd, 1960, p. 167, avait envisagé les deux hypothèses.

42. Voir l'article de N. Balcar,

J. Langlois, Y. Vandenberghe dans le présent volume.

43. Le musée conserve notamment une photo de l'œuvre en 1904, lors de son achat par Warocqué; voir Bourgeois à paraître.

44. Voir l'article suivant de N. Balcar,

J. Langlois, Y. Vandenberghe.

45. Voir par exemple les méthodes préconisées par Flinders Petrie, Methods and aims in archaeology, Londres, 1904, et F.

Rathgen, Die Konservirung von Alterthumsfunden, Berlin, 1898

46. B. Bourgeois, A. Pasquier, Le Gladiateur Borghèse et sa restauration, Paris, 1997, p. 15-16.

47. Autre cas sur la Muse pensive de Dresde, voir Liverani, 2014.

48. Hypothèse formulée par Tefnin, 1969, et largement reprise depuis lors. Sur l'inscription, E. Bernand, Inscriptions grecques d'Hermoupolis Magna et de sa nécropole,

Le Caire, 1999, p. 6-7.

49. Queyrel, 1984, p. 289.

50. Voir son article dans le présent volume.

51. Didi-Huberman G., 1998.

52. Lichtenstein, 2003, p. 158 sq, à propos de Baudelaire dans les Fleurs du mal.

\section{Bibliographie sélective}

Bloch Marc, 2007, Apologie pour l'histoire ou Métier d'historien, Paris, Armand Colin.

Blume C., 2014, "Bright Pink Blue and other Preferences" dans J. S. Østergaard and A.M. Nielsen (eds.), Transformations: Classical Sculpture in Colour, $\mathrm{Ny}$ Carlsberg Glyptotek, Copenhagen, p. 166-189.

Bourgeois (à paraître), «Ganôsis et réfections antiques de polychromie. Enquête sur le portrait en marbre «de Bérénice II » au Musée royal de Mariemont ", Cahiers de Mariemont.

Bourgeois B., Jockey Ph., 2010, «The Polychromy of Hellenistic Marble Sculpture in Delos » dans Brinkmann V., Primavesi O., Hollein M., (ed.), CIRCUMLITIO. The Polychromy of Antique and Mediaeval Sculpture, Liebieghaus, Frankfurt am Main, Hirmer Verlag, Munich, p. 224-239.

Bourgeois B., Jockey Ph., 2007, «Le marbre, l'or et la couleur» dans DescampsLequime S., (ed), Peinture et couleur dans le monde grec antique, Louvre, Paris, 5 Continents, Milan, p. 162-191.

Bourgeois B., Jockey Ph., 2005, «La dorure des marbres grecs. Nouvelle enquête sur la sculpture hellénistique de Délos ", Journal des Savants, juilletdécembre, p. 253-316.

Braustein Danièle, 1995, «En restaurant le Torse de Milet: de la technique à l'histoire ", Histoire de l'Art, n' 32 , De la restauration à l'histoire de l'art, p. 25-32.

Brecoulaki H., 2014, "Precious colours in Ancient Greek polychromy and painting: material aspects and symbolic value", Revue archéologique, $1, \mathrm{n}^{\circ} 57$, p. 3-35.

Brecoulaki à paraître, "Technical Studies in Ancient Greek Painting and Polychromy: from Scientific Investigation to Archaeological Interpretation" dans Guimier-Sorbets A.-M., Michailidis D. (dir.), Interior Decoration in the Eastern Mediterranean, during the Hellenistic and Roman times, Narnia Training Course, Nicosie, Paris.

Brinkmann V., Primavesi O., Hollein M., (ed.), 2010, CIRCUMLITIO. The Polychromy of Antique and Medieval Sculpture, Liebieghaus Skulpturensammlung, Frankfurt am Main.

Brinkmann V., Wünsche R. (ed.), 2003, Bunte Götter: die Farbigkeit antiker Skulptur, München, Glyptothek.

De Cesare M., 1997, Le statue in immagine: studi sulle raffigurazioni di statue nella pittura vascolare greca, Rome, "L'Erma» di Bretschneider.

Didi-Huberman G., 1998, «Viscosités et survivances. L'histoire de l'art à l'épreuve du matériau ", Critique, n 611, p. 138-162.

Elsner G., Knoll K. (herausg.), 1994, Das Albertinum vor 100 Jahren. DieSkulptursammlung Georg Treus: zur Erinnerung an die Eröffnung der
Sammlung der Originalbildwerke am 22. Dezember 1894, Dresde.

Graeve V. von, Preusser F., 1981, «Zur Technik griechischer Malerei auf Marmor ", JDAI, 96, p. 120-156.

Hellmann M.-Ch., 1992, Recherches sur le vocabulaire de l'architecture grecque d'après les inscriptions de Délos, BEFAR fasc. 278, EFA, Paris, éditions de Boccard.

Jockey Ph., 2013, Le mythe de la Grèce blanche. Histoire d'un rêve occidental, Belin.

Jockey Ph., 1998, «La technique composite à Délos à l'époque hellénistique» dans M. Schvoerer (ed.), Archéomatériaux. Marbres et autres roches. Actes de la IVe Conférence internationale ASMOSIA IV, Bordeaux, p. 305-316.

Kakoulli I., 2009, Greek Painting Techniques and Materials from the fourth to the first century $B C$, Archetype Publications, Londres.

Kyrieleis H., 1975, Bildnisse der Ptolemäer, Berlin.

Lichtenstein J., La tache aveugle. Essai sur les relations de la peinture et de la sculpture à l'âge moderne, Gallimard, Paris, 2003.

Liverani P., 2014, «Per una "Storia del colore". La policromia della scultura romana, un bilancio e qualche prospettiva» dans Liverani P., Santamaria U., (a cura di), Diversamente bianco: la policromia della scultura romana, Rome, éd. Quasar, p. 123-146.

Marcadé J., 1969, Au musée de Délos. Étude sur la sculpture hellénistique en ronde-bosse découverte dans l'île, BEFAR fasc. 215, EFA, Paris, éditions de Boccard.

Marconi C., 2011, "The birth of an image. The painting of a statue of Heracles and theories of representation in ancient Greek culture", Res 59/60, spring/autumn 2011, anthropology and aesthetics, p. 145-166.

Palagia O., 2006, "Marble carving techniques" dans Palagia O. (ed.), Greek Sculpture. Function, Materials, and Techniques in the Archaic and Classical Periods, Cambridge University Press, p. 243-279.

Pasquier A., 2007, «Les Aphrodites de Praxitèle» dans A. Pasquier, J.-L. Martinez (dir.), Praxitèle, Paris, Musée du Louvre éditions-Somogy.

Primavesi O., 2008, «Antike Dichter und Philosophen über die Farbigkeit der Skulptur» dans Brinkmann V. (ed.), Bunte Götter: die Farbigkeit antiker Skulptur, Liebieghaus Skulpturensammlung, Frankfurt am Main, p. 30-41.

Queyrel F., 1984, «Portraits de souverains lagides à Pompéi et à Délos ", $B C H 108$, p. 267-300.

Treu G., 1892, "Erwerbungen der Antikensammlungen in Deutschland. Dresden 1891", Arch. Anzeiger, VII, p. 158-159.

Tefnin R., 1969, «Un portrait de la reine Bérénice II trouvé en Égypte», L'Antiquité classique, t. XXXVIII, $1^{\text {er }}$ fasc., p. 89-100 et pl. I-IV. 